\title{
ANÁLISE DO POTENCIAL DE GIBBS-DONNAN VIA EQUAÇÃO DE POISSON-BOLTZMANN NA PRESENÇA DE CARGA FIXA
}

\author{
N. S. V. BARBOSA ${ }^{1}$, E. R. A. LIMA ${ }^{1}$ e F. W. TAVARES ${ }^{2,3}$ \\ ${ }^{1}$ Universidade do Estado do Rio de Janeiro, Programa de Pós-graduação em Engenharia \\ Química \\ ${ }^{2}$ Universidade Federal do Rio de Janeiro, Escola de Química, Escola de Química \\ ${ }^{3}$ Universidade Federal do Rio de Janeiro, Escola de Química, PEQ/COPPE \\ E-mail para contato: nathalia.vernin@gmail.com
}

\begin{abstract}
RESUMO - O potencial de Gibbs-Donnan aparece quando íons não permeáveis são desigualmente distribuídos entre duas soluções eletrolíticas separadas por uma membrana de permeabilidade seletiva, que permite a passagem de certos íons livremente entre as duas soluções. Essa distribuição de íons deve obedecer às leis da termodinâmica e ao princípio da eletroneutralidade. O objetivo deste trabalho é utilizar a equação de Poisson-Boltzmann (EPB) levando em conta as cargas móveis (íons) e as cargas fixas (proteínas localizadas, por exemplo) simultaneamente nos dois lados da membrana, calculando, além das concentrações iônicas, o potencial transmembrana. Os resultados mostram que a solução obtida da equação de Poisson-Boltzmann é consistente e concordante com o potencial de Gibbs-Donnan calculado pela equação de Nernst.
\end{abstract}

\section{INTRODUÇÃO}

O potencial de Gibbs-Donnan ocorre quando íons não permeáveis são desigualmente distribuídos entre duas soluções eletrolíticas separadas por uma membrana de permeabilidade seletiva, que permite a passagem de certos íons entre as duas soluções. A distribuição de íons obedece às leis da termodinâmica e ao princípio da eletroneutralidade entre os dois compartimentos ( $\alpha$ e $\beta$ ) separados pela membrana (KRYSINSKI; TIEN, 1986).

Para obter a diferença de potencial entre os dois compartimentos Prausnitz, Lichtenthaler e Azevedo (1999) abordam a igualdade de potencial químico do solvente entre os dois compartimentos, assim como a igualdade de potencial químico do sal entre os referidos compartimentos. Estes autores ressaltam que a igualdade do potencial químico referente a cada íon é incorreta. Haynie (2008) defende a mesma abordagem. Porém, na literatura, encontram-se controvérsias a respeito do assunto. Krysinski e Tien (1986), Sperelakis (2001) e Mafé et al.(1993) relatam a igualdade de potencial químico de cada espécie iônica e não do sal.

Ambos os caminhos convergem para uma equação que relaciona as concentrações de equilíbrio dos eletrólitos presentes em cada compartimento.

De acordo com a abordagem de Prausnitz, Lichtenthaler e Azevedo (1999), o potencial 
elétrico através da membrana pode ser calculado pela equação de Nernst:

$$
\Delta \psi=\frac{R T}{F z_{i}} \ln \frac{a_{\mathrm{i}}^{\beta}}{a_{\mathrm{i}}^{\alpha}}
$$

sendo $\Delta \psi$ a diferença de potencial transmembrana, $R$ a constante universal dos gases, $T$ a temperatura, $F$ a constante de Faraday, $z_{i}$ a valência do íon $i$ e $a_{i}$ a atividade do íon $i$. É comum considerar a atividade do íon $i$ igual à concentração deste.

Controversamente, Krysinski e Tien (1986) igualam o potencial químico dos íons, considerando que o desenvolvimento de um diferencial de pressão não afeta significativamente a distribuição de soluto. Independentemente da abordagem utilizada, ambos os desenvolvimentos conduzem à diferença de potencial calculado pela equação de Nernst.

O potencial de Gibbs-Donnan contribui para o potencial de membrana e partição iônica observados em células biológicas. Nestes sistemas, as macromoléculas presentes no meio citoplasmático são consideradas como polieletrólitos impermeáveis à membrana plasmática.

O objetivo deste trabalho consiste em resolver a equação de Poisson-Boltzmann (EPB), com a inclusão da densidade volumétrica de cargas fixas (polieletrólitos), para os dois meios simultaneamente e obter perfis de concentração dos íons e a diferença de potencial transmembrana. Uma questão importante é mostrar que a solução da EPB é consistente com o potencial transmembrana obtido por meio da equação de Nernst.

\section{TEORIA}

\subsection{Equação de Poisson-Boltzmann}

A equação de Poisson-Boltzmann é uma equação diferencial parcial de segunda ordem. Em sua forma clássica, é obtida a partir da equação de Poisson ao se considerar que a concentração de íons varia com a distância em relação à superfície de acordo com a distribuição de Boltzmann, sendo afetada pelo potencial eletrostático e pela temperatura, conforme abordagem de Gouy-Chapman para dupla camada elétrica.

A distribuição de Boltzmann pode ser descrita por

$$
c_{i}=c_{i, \infty} \exp \left(\frac{-e z_{i} \psi}{k_{B} T}\right)
$$

em que $c_{i}$ é a concentração do íon $i$ (número de íons por unidade de volume), $c_{i, \infty}$ é a concentração do íon $i$ a uma distância infinitamente grande da fase sólida (no seio do fluido, $\left.\psi_{\infty}=0\right)$, e é a carga do elétron, $z_{i}$ é a valência do íon $i, \psi$ é o potencial eletrostático, $k_{B}$ é a constante de Boltzmann e $T$ é a temperatura absoluta.

O produto $e z_{i} \psi$ representa o trabalho necessário para transferir um íon do seio da solução $\left(\psi_{\infty}=0\right)$ para o ponto em que o potencial é igual a $\psi$; o produto $k_{B} T$ representa a 
energia térmica (LIMA, 2008a).

A equação de Poisson, que relaciona a densidade volumétrica de carga $\rho_{l}$ com o potencial eletrostático $\psi$, pode ser escrita da seguinte forma:

$$
\varepsilon_{0} \nabla \cdot(\varepsilon \nabla \psi)=-\rho_{l}
$$

sendo $\varepsilon_{0}$ a permissividade do vácuo e $\varepsilon$ a constante dielétrica do meio.

A constante dielétrica do meio $(\varepsilon)$ pode ser considerada uniforme, como na teoria de Gouy-Chapman, ou função da posição no espaço, em uma abordagem mais realística (LIMA, 2008a, b, c, d).

Quando não há cargas elétricas envolvidas, o lado direito da Equação (33 anula-se, fazendo com que a equação de Poisson reduza-se a equação de Laplace. Havendo cargas elétricas envolvidas, a densidade volumétrica de carga $\rho_{l}$ é expressa pelo somatório das densidades volumétricas de carga móveis $\left(\rho_{m}\right)$ e fixas $\left(\rho_{f}\right)$ :

$$
\begin{aligned}
& \rho_{l}=\rho_{m}+\rho_{f} \\
& \rho_{m}=e \sum_{i} z_{i} c_{i} \\
& \rho_{l}=e \sum_{i} z_{i} c_{i}+\rho_{f}
\end{aligned}
$$

Substituindo a Equação 6 na equação de Poisson (Equação 3), e utilizando a distribuição de Boltzmann (Equação 2), para expressar a concentração de íons, obtém-se a equação de Poisson-Boltzmann, dada por:

$$
\varepsilon_{0} \nabla \cdot(\varepsilon \nabla \psi)=-e \sum_{i} z_{i} c_{i, \infty} \exp \left(\frac{-e z_{i} \psi}{k_{B} T}\right)-\rho_{f}
$$

As condições de contorno comumente associadas à Equação 7 podem ser de dois tipos: potencial especificado na superfície ou densidade superficial de carga $\sigma$ especificada na superfície. Neste trabalho, especificou-se a densidade superficial de carga em $x \rightarrow \infty$ e $x \rightarrow-\infty$ sendo iguais a 0, conforme as Equações (8) e (9).

$$
\begin{aligned}
& \left.\nabla \psi(x)\right|_{x \rightarrow \infty}=0 \\
& \left.\nabla \psi(x)\right|_{x \rightarrow-\infty}=0
\end{aligned}
$$

A equação de Poisson-Boltzmann foi adimensionada e resolvida por meio do método dos elementos finitos com aproximação em spline de segunda ordem em um sistema estacionário e unidimensional em coordenadas cartesianas (BARBOSA, 2014). 


\subsection{Densidade Volumétrica de Carga Fixa}

Utilizou-se uma função de regularização para expressar a densidade volumétrica de carga fixa entre os dois compartimentos separados pela membrana de permeabilidade seletiva, de forma a garantir a continuidade e eliminar possíveis problemas numéricos (BARBOSA, 2014).

Funções de regularização são usadas para unir duas funções distintas de uma mesma variável independente. Essa metodologia é importante quando se deseja utilizar a equação de Poisson-Boltzmann simultaneamente para os dois meios, em que se podem obter respostas um pouco diferentes em cada compartimento. A função de regularização serve para gerar uma função contínua em todo o intervalo da referida variável (FREITAS et al., 2012).

A função de regularização utilizada aqui consiste na função tangente hiperbólica. Sejam duas funções genéricas $g(x)$ e $h(x)$ de tal forma que:

$$
f(x)= \begin{cases}g(x), & \operatorname{para} x \leq x^{*} \\ h(x), & \operatorname{para} x>x^{*}\end{cases}
$$

A função $f(x)$ é descontínua. Para compatibilizar as duas soluções e transformá-la em uma função contínua $F(x)$, realiza-se o seguinte equacionamento:

$$
F(x)=v(x) h(x)+[1-v(x)] g(x)
$$

sendo:

$$
v(x)=\frac{1+\tanh \left(\frac{x-x^{*}}{\eta}\right)}{2}
$$

O parâmetro $\eta$ relaciona-se a suavidade da curva. Altos valores de $\eta$ geram curvas mais suaves, enquanto que baixos valores geram curvas mais bruscas.

\section{RESULTADOS}

Por meio da abordagem de Krysinski e Tien (1986), Sperelakis (2001) e Mafé et al. (1993) torna-se possível a utilização da distribuição de Boltzmann para descrever o perfil de concentração ao longo de uma solução eletrolítica, uma vez que nestas abordagens há uma igualdade de potencial químico dos íons entre as duas fases separadas pela membrana semipermeável. A utilização da distribuição de Boltzmann (Equação 2) vem da imposição de que o potencial químico de cada íon deve ser invariante com a distância.

A equação de Poisson-Boltzmann clássica unidimensional em coordenadas cartesianas na presença de cargas fixas foi resolvida a fim de comparar com os resultados obtidos pelo equilíbrio de Gibbs-Donnan. Em todas as situações analisadas nesta seção, o meio consiste em uma solução aquosa com eletrólitos a temperatura de $310,15 \mathrm{~K}$ e constante dielétrica do meio igual a 74,16. Além disso, a membrana semipermeável não apresenta carga.

O primeiro caso analisado refere-se a um sistema eletrolítico com $\mathrm{NaCl}$. No equilíbrio, 
em um dos compartimentos há uma concentração equivalente constante de proteína (com carga líquida negativa) igual a $100 \mathrm{mEq} / \mathrm{L}$, enquanto que no outro compartimento a concentração no seio da fase fluida é igual a $70 \mathrm{mM}$ de $\mathrm{Na}^{+}$e $70 \mathrm{mM}$ de $\mathrm{Cl}^{-}$. O perfil de densidade volumétrica de carga fixa em $\mathrm{MCm}^{-3}$ pode ser observado na Figura 1. Para a construção da função de regularização de densidade volumétrica de carga fixa utilizou-se valor de $\eta$ igual a 0,1 .

De acordo com o princípio da eletroneutralidade e leis da termodinâmica, as concentrações de $\mathrm{Na}^{+}$e $\mathrm{Cl}^{-}$no meio que contém a proteína são respectivamente iguais a 136,02 mM e 36,02 mM. Segundo a equação de Nernst, o potencial de Gibbs-Donann entre os dois compartimentos separados pela membrana é igual a $-17,754 \mathrm{mV}$.

Por meio da equação de Poisson-Boltzmann, obteve-se uma diferença de potencial entre os seios das fases fluidas igual ao previsto pelo potencial de Gibbs-Donnan (Figura 1B); o mesmo fato ocorreu em relação às concentrações de $\mathrm{Na}^{+}$e $\mathrm{Cl}^{-}$no seio da fase fluida do compartimento a esquerda (Figura 1C). A membrana é considerada de espessura infinitesimal.

A

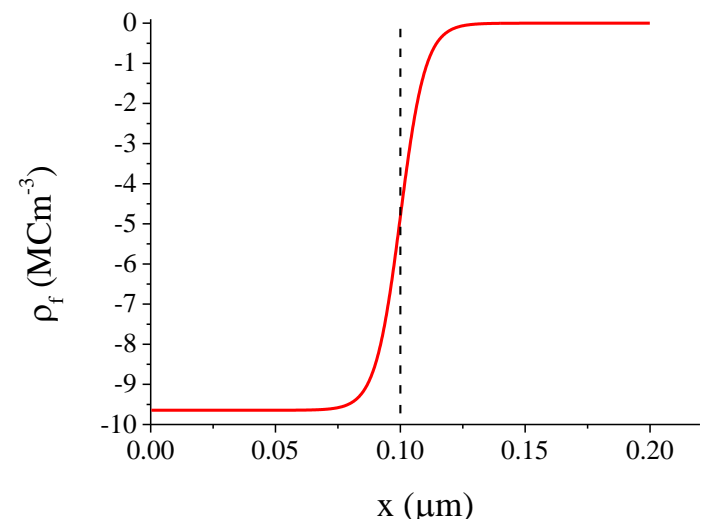

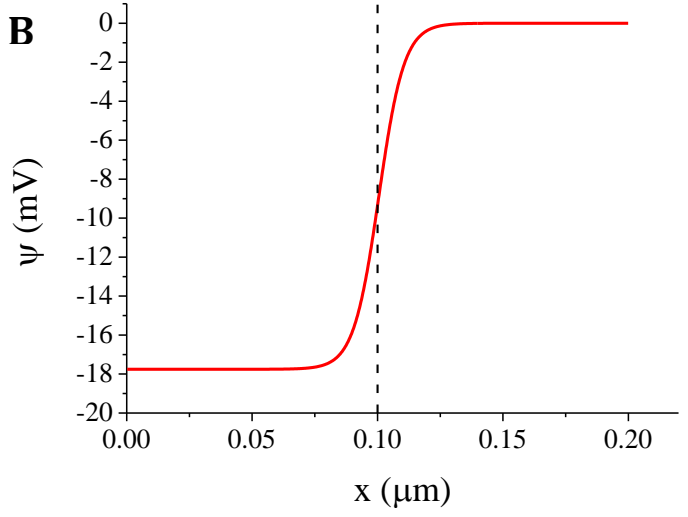

C

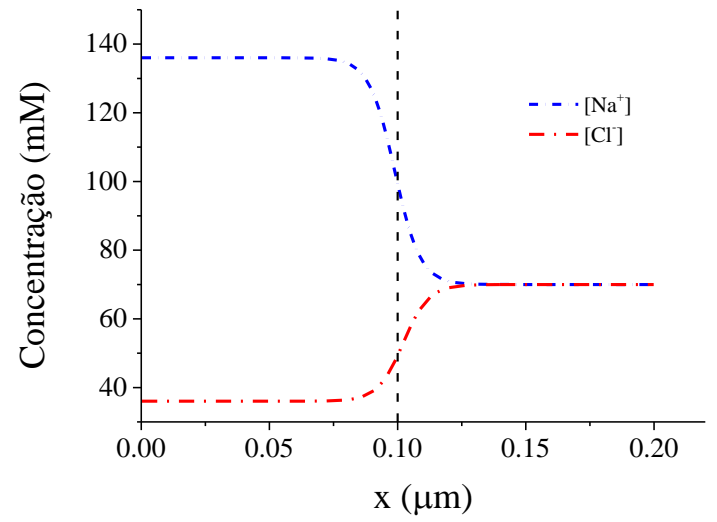

Figura 1 - Perfil de densidade volumétrica de carga fixa (A), potencial elétrico (B) e concentrações de $\mathrm{Na}^{+}$e $\mathrm{Cl}^{-}(\mathrm{C})$ nas proximidades de uma membrana semipermeável. A linha tracejada preta em $0.1 \mu \mathrm{m}$ refere-se à localização da membrana. O compartimento da esquerda possui uma concentração equivalente constante de proteínas igual a $100 \mathrm{mEq} / \mathrm{L}$ no seio da fase fluida, enquanto que no outro compartimento a concentração no seio da fase fluida é igual a $70 \mathrm{mM} \mathrm{NaCl}$. 
O segundo caso analisado consiste no compartimento à esquerda apresentando $500 \mathrm{mM}$ de um cátion impermeável à membrana e o compartimento à direita apresentando concentração de $340 \mathrm{mM}$ do sal $\mathrm{NaCl}$. Segundo o equilíbrio de Gibbs-Donnan, o potencial elétrico entre os dois compartimentos é igual a $18,209 \mathrm{mV}$ e as concentrações de $\mathrm{Na}^{+} \mathrm{e} \mathrm{Cl}^{-}$no seio da fase fluida do compartimento à esquerda são iguais a $172,02 \mathrm{mM}$ e $672,02 \mathrm{mM}$, respectivamente.

Para a construção do perfil de densidade volumétrica de carga fixa utilizou-se a função de regularização com valor de $\eta=0,1$.

A solução da equação de Poisson-Boltzmann com carga fixa forneceu os mesmos valores para o potencial elétrico e concentração dos íons $\mathrm{Na}^{+} \mathrm{e}^{-} \mathrm{Cl}^{-}$no seio da fase fluida do compartimento a esquerda, como pode ser observado na Figura 2.

A

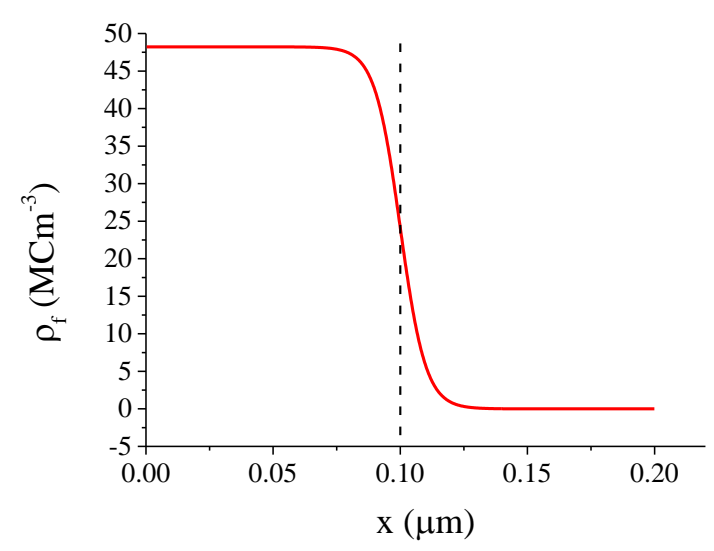

B

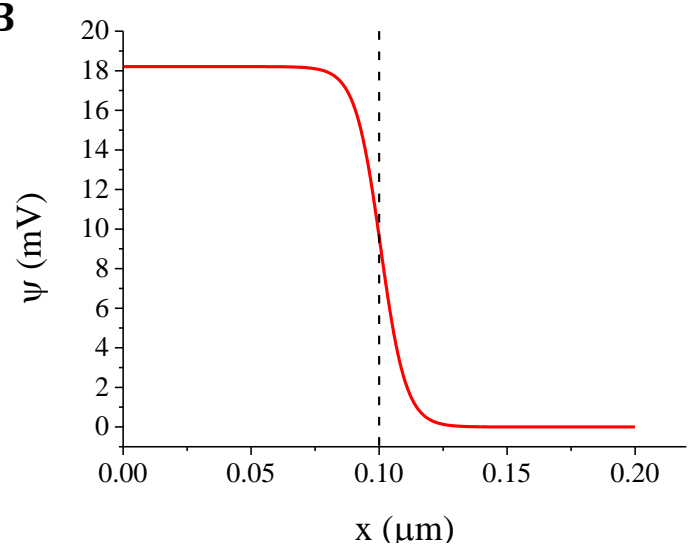

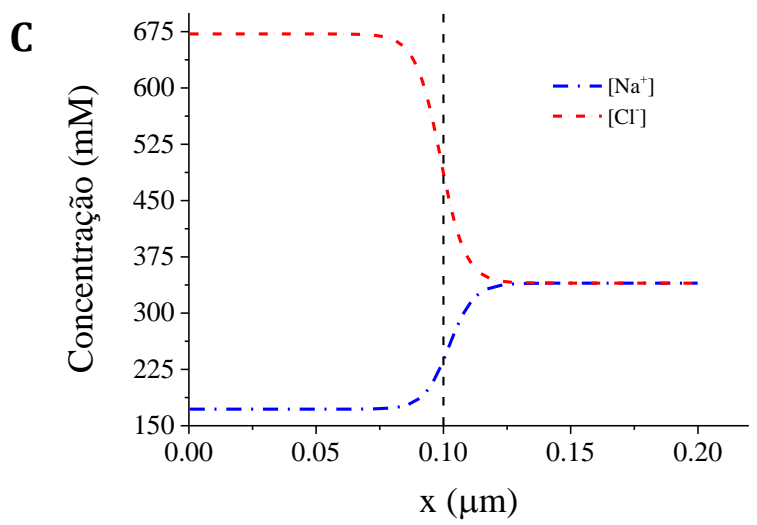

Figura 2 - Perfil de densidade volumétrica de carga fixa (A), potencial elétrico (B) e concentrações de $\mathrm{Na}^{+}$e $\mathrm{Cl}^{-}(\mathrm{C})$ na presença de uma membrana semipermeável na qual o compartimento a esquerda possui uma concentração de cátion impermeável igual a $500 \mathrm{mM} \mathrm{e}$ concentrações de $\mathrm{Na}^{+}$e Cl${ }^{-}$iguais a $172,02 \mathrm{mM}$ e $672,02 \mathrm{mM}$, respetivamente, no seio da fase fluida, e o compartimento a direita possui concentrações no seio da fase fluida iguais a 340 $\mathrm{mM}$ de $\mathrm{NaCl}$. A linha tracejada preta em $0.1 \mu \mathrm{m}$ refere-se à localização da membrana. 
O terceiro caso consiste na presença de proteína em um dos compartimentos, com densidade volumétrica de carga fixa igual a $-4,34 \mathrm{MCm}^{-3}$ (concentração equivalente igual a 45 $\mathrm{mEq}$ ) no seio da fase fluida na presença dos íons $\mathrm{Na}^{+}, \mathrm{K}^{+}$e $\mathrm{Cl}^{-}$A concentração desses íons no seio da fase fluida ausente de proteína é igual a $140 \mathrm{mM}, 10 \mathrm{mM}$ e $150 \mathrm{mM}$, respectivamente.

Segundo a teoria do equilíbrio de Gibbs-Donnan, o potencial elétrico entre os dois compartimento no terceiro caso é igual a $-3,99 \mathrm{mV}$ e as concentrações de $\mathrm{Na}^{+}, \mathrm{K}^{+}$e $\mathrm{Cl}^{-}$no seio da fase fluida referente ao compartimento com proteína são iguais a 162,57 mM, 11,61 $\mathrm{mM}$ e $129,18 \mathrm{mM}$.

Assim como nos casos anteriores, obteve-se o mesmo resultado para a diferença de potencial e concentrações de íons no seio da fase fluida do compartimento que contém o íon impermeável através da resolução da equação de Poisson-Boltzmann (Erro! Fonte de referência não encontrada.).

A

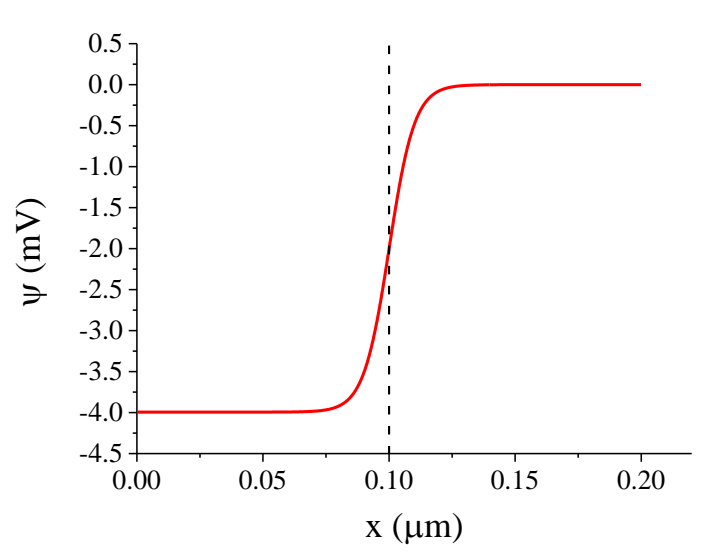

B

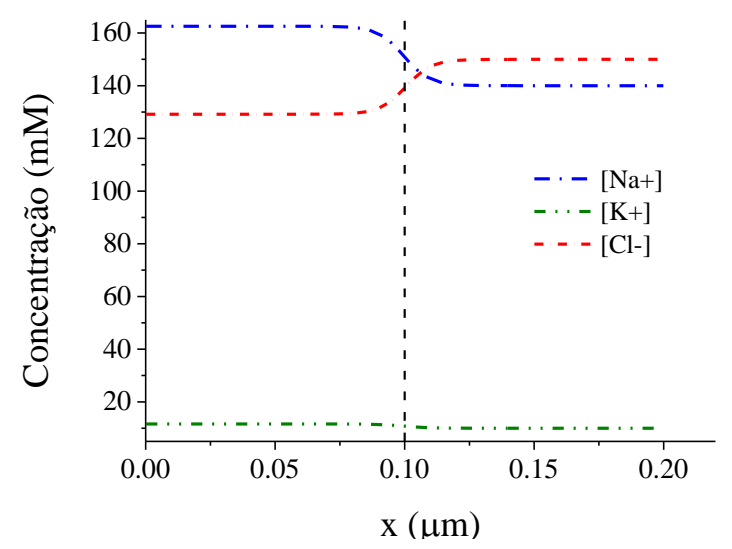

Figura 3 - Perfil do potencial elétrico (A) e concentrações de $\mathrm{Na}^{+}, \mathrm{K}^{+}$e $\mathrm{Cl}^{-}$(B) na presença de uma membrana semipermeável, na qual o compartimento a esquerda possui uma

concentração equivalente de proteína impermeável igual a $45 \mathrm{mEq}$ e o compartimento a direita possui concentrações no seio da fase fluida iguais a $140 \mathrm{mM} \mathrm{de} \mathrm{Na}^{+}, 10 \mathrm{mM}$ de $\mathrm{K}^{+} \mathrm{e}$

$150 \mathrm{mM}$ de $\mathrm{Cl}^{-}$. A linha tracejada preta em $0.1 \mu \mathrm{m}$ refere-se à localização da membrana.

A linha tracejada preta refere-se à localização da membrana semipermeável.

Dessa forma, é possível observar que o potencial de Gibbs-Donnan (diferença de potencial transmembrana) surge automaticamente da resolução da equação de PoissonBoltzmann e, desta forma, não precisa ser imposta para tratar sistemas carregados e contendo membranas.

\section{CONCLUSÃO}

Com base nos resultados obtidos, conclui-se que a equação de Poisson-Boltzmann com a inclusão de carga fixa pode ser uma ferramenta útil para analisar potenciais e concentrações iônicas de sistemas contendo membranas de permeabilidade seletiva, típicas de membranas biológicas. Essas membranas apresentam potencial de Gibbs-Donnan (diferença de potencial transmembrana) que surge automaticamente da resolução da equação de Poisson-Boltzmann, 
conforme mostrado aqui.

\section{REFERÊNCIAS}

BARBOSA, N. S. V. Aplicação da equação de Poisson-Boltzmann modificada em sistemas biológicos: análise da partição iônica em um eritrócito. 2014. 149 f. Dissertação (Mestrado em Engenharia Química) - Programa de Pós-graduação em Engenharia Química, Universidade do Estado do Rio de Janeiro, Rio de Janeiro, 2014.

FREITAS, T. C.; QUINTO, T. C.; SECCHI, A. R.; BISCAIA, E. C. An Efficient adjoint-free dynamic optimization methodology for batch processing using Pontryagin's formulation. In: BOGLE, I. D. L.; FAIRWEATHER, M. (Ed.) 22nd European Symposium on Computer Aided Process Engineering. Amsterdam: Elsevier, 2012.

HAYNIE, D. T. Biological Thermodynamics. 2. ed. New York: Cambridge University Press, 2008.

KRYSINSKI, P.; TIEN, H. T. Membrane electrochemistry. Prog. Surf. Sci., v. 23, n. 4, p. 317-412, 1986.

LIMA, E. R. A. Cálculo de Propriedades físico-químicas de sistemas coloidais via equação de Poisson-Boltzmann. 2008. 144 f. Tese (Doutorado em Engenharia Química) Programa em Pós-Graduação em Engenharia Química, COPPE, Universidade Federal do Rio de Janeiro, Rio de Janeiro, 2008a.

LIMA, E. R. A.; BOSTRÖM, M.; HORINEK, D.; BISCAIA, E. C.; KUNZ, W.; TAVARES, F. W. Co-ion and ion competition effects: ion distributions close to a hydrophobic solid surface in mixed electrolyte solutions. Langmuir, v. 24, n. 8, p. 3944-3948, 2008 b.

LIMA, E. R. A.; BOSTRÖM, M.; SERNELIUS, B. E.; HORINEK, D.; NETZ, R. R.; BISCAIA, E. C.; KUNZ, W.; TAVARES, F. W. Forces between air-bubbles in electrolyte solution. Chem. Phys. Lett., v. 458, n. 4-6, p. 299-302, 2008 c.

LIMA, E. R. A; HORINEK, D.; NETZ, R. R.; BISCAIA, E. C.; TAVARES, F. W.; KUNZ, W.; BOSTRÖM, M. Specific ion adsorption and surface forces in colloid science. $J$. Phys. Chem. B, v. 112, n. 6, p. 1580-1585, 2008 d.

MAFÉ, S.; MANZANARES, J. A.; REISS, H. Donnan phenomena in membranes with charge due to ion adsorption. Effects of the interaction between adsorbed charged groups. $J$. Chem. Phys., v. 98, n. 3, p. 2325-2331, 1993.

PRAUSNITZ, J. M.; LICHTENTHALER, R. N.; AZEVEDO, E. G. Molecular thermodynamics of fluid-phase equilibria. 3. ed. Upper Saddle River: Prentice Hall, 1999.

SPERELAKIS, N. Cell Physiology Source Book: A molecular Approach. 3. ed. Ohio: Academic Press, 2001. 